\title{
Cauchy and Nonlocal Multi-Point Problems for Distributed Order Pseudo-Differential Equations, Part One
}

\author{
Sabir Umarov and Rudolf Gorenflo
}

\begin{abstract}
We treat the question of existence, uniqueness and construction of a solution to the Cauchy and multi-point problems for a general linear evolution equation with (in general) temporal fractional derivatives with distributed orders. Such equations have met great interest in recent years among researchers in viscoelasticity and in anomalous diffusion processes, and there are numerical analysts who consider them as a challenge. So, we find it desirable to put their theory on a strong and general mathematical basis. After an outline of relevant function spaces and the duality structure generated by them we treat, by Laplace-Fourier techniques, first the Cauchy problem, then the general multi-point problem (where the values of linear combinations of the unknown solution at different instants of time are prescribed). We condense our results in theorems on strong and on weak solutions.
\end{abstract}

Keywords: Distributed order fractional differential equation, pseudo-differential operator, Cauchy problem, multi-point value problem, Caputo fractional derivative

MSC 2000: Primary 26A33, secondary 45K05, 35A05, 35S10, 35S15

\section{Introduction}

This paper is devoted to distributed order fractional differential equations. In recent years such equations have met interest of several researchers (see, for example [2], [5]- [8], [22], [30] and references therein) who have treated relevant physical and numerical problems, mainly of evolutionary character. Our main purpose here is the mathematical treatment of such equations. We will study the solvability and uniqueness issues of initial and some boundary value problems

Sabir Umarov: Computer Technologies Department, National University of Uzbekistan, Tashkent, Uzbekistan; sabir0725@yahoo.com

Rudolf Gorenflo: Fachbereich Mathematik und Informatik, Freie Universität Berlin, Berlin, Germany; gorenflo@mi.fu-berlin.de 
for them in different function spaces. The general formulation of the problems can be settled in the following framework: introduction of the distributed timefractional differential operator equation and study of the Cauchy and some other boundary value problems for it.

Our distributed order time fractional differential-operator equation has the general form

$$
\int_{0}^{m} A(r) D_{*}^{r} u(t) d r=B u(t), \quad t>0
$$

where $A(r)$ (for a fixed $r \in[0, m]$ ) and $B$ are linear closed operators defined in a certain locally convex topological vector space $X$, the function $u(t)$ is unknown and belongs to the space $C^{(m)}(0, T ; X)$ with some $T>0$ and $D_{*}^{r}$ is the operator of fractional differentiation of order $r$ in the Caputo sense (see, for example, [4], $[12],[27])$

$$
\begin{aligned}
D_{*}^{r} f(t) & =\frac{1}{\Gamma(m-r)} \int_{0}^{t}(t-\tau)^{m-r-1} f^{(m)}(\tau) d \tau, \quad t>0, m-1<r<m \\
D_{*}^{m} f(t) & =f^{(m)}(t)
\end{aligned}
$$

for $m \in \mathbf{N}$. By $\mathbf{N}$ we mean, as usual, the set of positive integers. An essential distinctive feature of this model is that integration in (1) is performed over the variable $r$, the order of differentiation. Such models arise in a natural way in the kinetic theory (see [7] and [30]) when the exact scaling is lacking or in the theory of elasticity [22] for description of rheological properties of composite materials (see also the examples below). We will not present here results for such general case but will restrict ourselves to considering some particular cases of $A(r), B$ and $X$, leaving the general case for a separate paper.

In this paper we will consider the following distributed order time-fractional differential equation with spatial pseudo-differential operators

$$
\int_{0}^{m} A(r ; D) D_{*}^{r} u(t, x) d r=B(D) u(t, x), \quad t>0, x \in \mathbb{R}^{n},
$$

with the Cauchy conditions

$$
\frac{\partial^{k} u(0, x)}{\partial t^{k}}=\varphi_{k}(x), \quad x \in \mathbb{R}^{n}, k=0, \ldots, m-1,
$$

where the $\varphi_{k}, k=0, \ldots, m-1$, are given functions in certain spaces described later, $D=\left(D_{1}, \ldots, D_{n}\right), D_{j}=-i \frac{\partial}{\partial x_{j}}, j=1, \ldots, n, A(r ; D)$ (for every fixed value of the parameter $r \in[0, m])$ and $B(D)$ are pseudo-differential operators with the symbols $A(r ; \xi)$ and $B(\xi)$, respectively, which are continuous functions of $\xi$ defined in an open domain $G \subset \mathbb{R}^{n}$. The pseudo-differential operators used in this paper are defined in Section 2 by the formula (9) below. Some 
spatial pseudo-differential operators frequently used to describe anomalous diffusion processes with infinite second moment of the spatially zero-centered fundamental solution also have continuous symbols. So, $A(r ; D)$ and $B(D)$ can be fractional order spatial operators as well.

For our further considerations we need some properties of pseudo-differential operators with symbols singular in dual variables. These operators were studied by Dubinskij [10], [11] in the case of analytic symbols defined in a domain $G$ and used by several authors including [32], [33].

In constructions we use in the present paper, in order to include operators with non-analytic symbols, we choose a direct approach for definition of such operators, which is distinct from the above mentioned and does not require glueing of local elements of pseudo-differential operators used in case of analytic symbols. This approach (see [16], [34], [35]) allows us to consider nonanalytic symbols which may have non-integrable or other type singularities on the boundary of $G$ or in its exterior.

We also outline briefly some results for solution of the more general multipoint value problem for equation (3) with the conditions

$$
\sum_{j=0}^{m-1} \Gamma_{k j}(D) \frac{\partial^{k} u\left(t_{k j}, x\right)}{\partial t^{k}}=\varphi_{k}(x), \quad k=0, \ldots, m-1, x \in \mathbb{R}^{n},
$$

where the $\Gamma_{k j}(D), k, j=0, \ldots, m-1$, are pseudo-differential operators whose symbols $\Gamma_{k j}(\xi), k, j=0, \ldots, m-1$, are continuous functions in $G, t_{k j} \in[0, T]$, $0<T<\infty$, and the $\varphi_{k}, k=0, \ldots, m-1$, are given functions. We note that the Cauchy problem (3), (4) is a particular case of the multi-point value problem (3), (5).

The equation (3) is a generalization of fractional/non-fractional differential equations and important from the viewpoint of applications. To justify this we consider some frequently used particular cases, putting definitions formally:

1. Let $A(r ; D)=\delta(r-\beta) I$ with the Dirac function (distribution) $\delta$ and the identity operator $I$. In this case we get the fractional differential equation

$$
D_{*}^{\beta} u(t, x)=B(D) u(t, x), \quad t>0, x \in \mathbb{R}^{n}, \beta>0 .
$$

\subsection{Time-fractional equation.}

Let $B(D)=\Delta$ be the Laplace operator with the symbol $B(\xi)=-|\xi|^{2}$. Then we have the time-fractional differential equation

$$
D_{*}^{\beta} u(t, x)=\Delta u(t, x), \quad t>0, x \in \mathbb{R}^{n}, \beta>0 .
$$

The Cauchy problem for this equation represents a fractional model of diffusion (sub-diffusion) in the case $0<\beta<1$, which has an important role in transport 
theory and random walk [23], [25], [26]. It represents processes intermediate between diffusion and wave propagation in the case $1<\beta<2$ [31]. The solution has the relaxation property in the case $0<\beta<1$ and the oscillation-relaxation property when $1<\beta<2[16]$.

\subsection{Space-fractional differential equation.}

Let $A(r ; D)=\delta(r-1) I$ and $B(D)=D_{0}^{\alpha}$, the latter being the operator with the symbol $-|\xi|^{\alpha}, \xi \in \mathbb{R}^{n}$, which is a continuous function. Then we come to the equation

$$
\frac{\partial u(t, x)}{\partial t}=D_{0}^{\alpha} u(t, x), \quad t>0, x \in \mathbb{R}^{n}, \alpha>0,
$$

which describes in the case $0<\alpha<2$ an anomalous (to use physical language) diffusion process with infinite second moment of its spatially zero-centered fundamental solution. We note that $D_{0}^{\alpha}$ is connected with the inversion of the fractional Riesz potential (see, for example, [29]) and can be written in the form

$$
D_{0}^{\alpha} f(x)=\frac{-1}{c_{n}(\alpha)} \int_{R^{n}} \frac{\left(\Delta_{h}^{2} f\right)(x)}{|h|^{n+\alpha}} d h .
$$

Here $\Delta_{h}^{2}$ is the second order finite difference with the spatial step $h \in \mathbb{R}^{n}$ and $c_{n}(\alpha)$ is a constant (for the values of this constant see [29]). In the onedimensional case and under the condition $0<\alpha \leq 2$ this equation describes symmetric Lévy-Feller diffusion processes, which are Markovian [13]. Symmetric and non-symmetric random walk models approximating Lévy-Feller diffusion processes were presented in detail by Gorenflo and Mainardi [13], [14], [15]. In the multidimensional case it is studied by Umarov and Gorenflo [36]. For more general operators on the right hand side of (7), including operators with variable orders, the corresponding Feller semigroups are constructed in [18][21]. These results allow to judge on existence of a solution (in some sense) to the corresponding Cauchy problem for such equations.

\subsection{Time- and space-fractional equation.}

Let $A(r ; D)=\delta(r-\beta) I, \beta \in(0,1)$ and $B(D)=D_{0}^{\alpha}$. Then we get the time-space fractional differential equation

$$
D_{*}^{\beta} u(t, x)=D_{0}^{\alpha} u(t, x), \quad t>0, x \in \mathbb{R}^{n}, \alpha, \beta>0,
$$

which describes anomalous diffusion processes, in particular such ones with non-Markovian character (see [17], [24], [26]).

2. The next example relates to sub-diffusion with retardation, studied in [7]. Let $A(r ; D)=b_{1} \delta\left(r-\beta_{1}\right) I+b_{2} \delta\left(r-\beta_{2}\right) I$, with $0<\beta_{1}<\beta_{2} \leq 1, b_{1}>$ $0, b_{2}>0, b_{1}+b_{2}=1$ and $B(D)=k \frac{\partial^{2}}{\partial x^{2}}(n=1), k=$ const $>0$. Then we get the equation

$$
b_{1} D_{*}^{\beta_{1}} u(t, x)+b_{2} D_{*}^{\beta_{2}} u(t, x)=k \frac{\partial^{2}}{\partial x^{2}} u(t, x)
$$


which describes a subdiffusion process with retardation. In [7] the Cauchy problem for the equation

$$
\int_{0}^{1} \tau^{\beta-1} w(\beta) \frac{\partial^{\beta} p}{\partial t^{\beta}} d \beta=k \frac{\partial^{2} p}{\partial x^{2}}
$$

referred to as the "normal form" of the distributed order fractional diffusion is also studied. Note that this equation corresponds to the case $A(r, \xi)=\tau^{r-1} w(r)$ with $\tau>0, w(r)>0$ and $B(D)=k \frac{\partial^{2}}{\partial x^{2}}$.

3. The authors of [22] have discussed equations of the form

$$
\int_{0}^{2} k(q) D^{q} y(t) d q+F(y)=f(t)
$$

which describe properties of composite materials. Note that $k(q), F(y)$ and $f(t)$ are given functions connected with different characteristics of viscoelastic and viscoinertial materials with rheological properties.

4. Let $A(r ; D))=\sum_{k=1}^{m} \delta\left(r-\beta_{k}\right) A_{k}(D)$ with $k-1<\beta_{k} \leq k, A_{k}(D), k=$ $0, \ldots, m-1$, be pseudo-differential operators with symbols $A_{k}(\xi)$ continuous in $G$. In this case we obtain the equation

$$
D_{*}^{\beta_{m}} u(t, x)+\sum_{k=1}^{m-1} A_{k}(D) D_{*}^{\beta_{k}} u(t, x)+A_{0}(D) u(t, x)=0, t \in(0, T), x \in \mathbb{R}^{n} .
$$

In the case $\beta_{k} \in \mathbb{N}, k=1, \ldots, m$, the Cauchy problem for this equation with analytic symbols or with symbols having singularities was studied, for example, by Dubinskij [10], Umarov [33] and Tran Duc Van [32]. Antipko and Borok [1], Borok [3], Ptashnik [28], and Umarov [34], [35] (see also references therein) considered multi-point value problems with integer $\alpha_{k}$. For fractional $\alpha_{k}$ the Cauchy and multi-point value problems are studied in [16].

Our paper is organized as follows. In Section 2 we will give briefly the description of the space $\Psi_{G, p}\left(\mathbb{R}^{n}\right)$ introduced in [34], define pseudo-differential operators with singular symbols and recall some of their properties referring the readers for details to [34], [16]. In Section 3 we will construct a general representation formula for the solution of the Cauchy problem for distributed order differential equations. In Section 4 we will study in detail properties of solution operators obtained in Section 3. Using these properties in Section 5 we will prove solvability theorems for the Cauchy problem (3), (4) in the space $\Psi_{G, p}\left(\mathbb{R}^{n}\right)$ and in its dual space $\left(\Psi_{G, p}\left(\mathbb{R}^{n}\right)\right)^{*}=\Psi_{-G, q}^{\prime}\left(\mathbb{R}^{n}\right), q=p /(p-1)$. Finally in Section 6 we will consider the general multi-point value problem (3), (5) and outline the related results. 


\section{Function spaces and pseudo-differential operators}

In this section we introduce some function spaces and briefly recall their essential properties to be used later for formulation of solvability theorems. For details see [34] and [16].

For a given open domain $G \subset \mathbb{R}^{n}$ let $f$ be a function in $L_{p}\left(\mathbb{R}^{n}\right), 1 \leq p \leq$ $\infty$, whose Fourier transform

$$
\hat{f}(\xi)=F f(\xi)=\int_{\mathbb{R}^{n}} f(x) e^{-i x \xi} d x
$$

has a compact support in $G$. The set of all such functions endowed with the convergence defined below is denoted by $\Psi_{G, p}\left(\mathbb{R}^{n}\right)$ : A sequence of functions $f_{m} \in \Psi_{G, p}\left(\mathbb{R}^{n}\right)$ is said to converge to an element $f_{0} \in \Psi_{G, p}\left(\mathbb{R}^{n}\right)$ iff:

(a) there exists a compact set $K \subset G$ such that $\operatorname{supp} \hat{f}_{m} \subset K$ for all $m \in \mathbf{N}$;

(b) $\left\|f_{m}-f_{0}\right\|_{p}=\left(\int_{R^{n}}\left|f_{m}-f_{0}\right|^{p} d x\right)^{\frac{1}{p}} \rightarrow 0$ for $m \rightarrow \infty$.

In the case $p=2$ we write simply $\Psi_{G}\left(\mathbb{R}^{n}\right)$ instead of $\Psi_{G, 2}\left(\mathbb{R}^{n}\right)$. According to the Paley-Wiener-Schwartz theorem, the elements of $\Psi_{G, p}\left(\mathbb{R}^{n}\right)$ are entire functions of exponential type which, restricted to $\mathbb{R}^{n}$, are in the space $L_{p}\left(\mathbb{R}^{n}\right)$.

Let $q=p /(p-1)$. Denote by $\Psi_{-G, q}^{\prime}\left(\mathbb{R}^{n}\right)$ the space of all linear bounded functionals defined on the space $\Psi_{G, p}\left(\mathbb{R}^{n}\right)$ endowed with the weak topology. Namely, we say that a sequence of functionals $g_{N} \in \Psi_{-G, q}^{\prime}\left(\mathbb{R}^{n}\right)$ converges to an element $g_{0} \in \Psi_{-G, q}^{\prime}\left(\mathbb{R}^{n}\right)$ in the weak sense if for all $f \in \Psi_{G, p}\left(\mathbb{R}^{n}\right)$ the sequence of numbers $\left\langle g_{N}, f\right\rangle$ converges to $\left\langle g_{0}, f\right\rangle$ as $N \rightarrow \infty$. By $\langle g, f\rangle$ we mean the value of the action of $g \in \Psi_{-G, q}^{\prime}\left(\mathbb{R}^{n}\right)$ on an element $f \in \Psi_{G, p}\left(\mathbb{R}^{n}\right)$.

Let $A(\xi)$ be defined and continuous in a domain $G \subset \mathbb{R}^{n}$. This function may have singularities of arbitrary type outside of $G$ or on its boundary. Define a pseudo-differential operator $A(D)$ with a symbol $A(\xi)$ on a function $\varphi \in$ $\Psi_{G, p}\left(\mathbb{R}^{n}\right)$ by the formula

$$
A(D) \varphi(x)=\frac{1}{(2 \pi)^{n}} \int_{G} A(\xi) F \varphi(\xi) e^{i x \xi} d \xi
$$

where $F \varphi$ is the Fourier transform of $\varphi$.

We define the operator $A(-D)$ acting in the space $\Psi_{-G, q}^{\prime}\left(\mathbb{R}^{n}\right)$ by the formula

$$
\langle A(-D) f, \varphi\rangle=\langle f, A(D) \varphi\rangle, \quad f \in \Psi_{-G, q}^{\prime}\left(\mathbb{R}^{n}\right), \varphi \in \Psi_{G, p}\left(\mathbb{R}^{n}\right) .
$$

We also recall the following statement (see Lemma 3 in [16] and Lemma 3 in [34]), which is important for proof of solvability theorems for the Cauchy and multipoint problems. 
Theorem 2.1. An arbitrary pseudo-differential operator $A(D)(A(-D))$ whose symbol $A(\xi)(A(-\xi))$ is continuous in a domain $G \subset \mathbb{R}^{n}\left(-G \subset \mathbb{R}^{n}\right)$ acts from the space $\Psi_{G, p}\left(\mathbb{R}^{n}\right)\left(\Psi_{-G, q}^{\prime}\left(\mathbb{R}^{n}\right)\right)$ into itself. Moreover, if $A(\xi) g(\xi)$ is a multiplier in $L_{p}$ for any $g \in C_{0}^{\infty}(G)$, then this operator acts continuously.

\section{Representation of the solution to the Cauchy problem for distributed order pseudo-differential equations}

In this section we will construct the representation formula for a solution of the Cauchy problem for the distributed order pseudo-differential equation

$$
\begin{aligned}
\int_{0}^{m} A(r ; D) D_{*}^{r} u(t, x) d r & =B(D) u(t, x), \quad t>0, x \in \mathbb{R}^{n} \\
\frac{\partial^{k} u(0, x)}{\partial t^{k}} & =\varphi_{k}(x), \quad x \in \mathbb{R}^{n}, k=0, \ldots, m-1 .
\end{aligned}
$$

First we find the formal representation of a solution and then, relying on this representation, study its properties.

We split the problem (11), (12) into $m$ Cauchy problems, one for each index $j \in\{0,1, \ldots, m-1\}$, keeping (11) and using the the Cauchy conditions

$$
\begin{gathered}
u(0, x)=0, \ldots, \frac{\partial^{j-1} u(0, x)}{\partial t^{j-1}}=0, \\
\frac{\partial^{j} u(0, x)}{\partial t^{j}}=\varphi_{j}(x), \\
\frac{\partial^{j+1} u(0, x)}{\partial t^{j+1}}=0, \ldots, \frac{\partial^{m-1} u(0, x)}{\partial t^{m-1}}=0 .
\end{gathered}
$$

If we denote by $u_{j}(t, x)$ a solution to $(11),(13)$, then the general solution to (11), (12) due to its linearity, is represented in the form

$$
u(t, x)=\sum_{j=0}^{m-1} u_{j}(t, x) .
$$

Applying formally the Fourier transform to $(11),(13)$ we get the $\xi$-parametrized equation

$$
\int_{0}^{m} A(r ; \xi) D_{*}^{r} \hat{u}(t, \xi) d r=B(\xi) \hat{u}(t, \xi)
$$

and the corresponding initial conditions

$$
\hat{u}(0, \xi)=0, \ldots, \hat{u}^{j-1}(0, \xi)=0,
$$




$$
\begin{gathered}
\hat{u}^{j}(0, \xi)=\hat{\varphi}_{j}(\xi), \\
\hat{u}^{j+1}(0, \xi)=0, \ldots, \hat{u}^{m-1}(0, x)=0 .
\end{gathered}
$$

We rewrite the left hand side of (14) in the form

$$
\int_{0}^{m} A(r ; \xi) D_{*}^{r} \hat{u}(t, \xi) d r=\sum_{k=1}^{m} \int_{k-1}^{k} A(r, \xi) D_{*}^{r} \hat{u}(t, \xi) d r
$$

and apply the Laplace transform to both sides. Taking $k-1<r<k$ into account and making use of the well known formula (see [12] and [27])

$$
L\left[D_{*}^{r} f(t)\right](s)=L[f](s) s^{r}-\sum_{l=0}^{k-1} f^{(l)}(0) s^{r-l-1},
$$

where $L$ stands for the Laplace transform (we will use on equal status the notations $\tilde{f}(s)$ and $L[f](s)$ for the Laplace transform of $f$ ), we have

$$
\begin{aligned}
\int_{k-1}^{k} A(r, \xi) L\left[D_{*}^{r} \hat{u}(t, \xi)\right] d r= & \tilde{\hat{u}}(s, \xi) \int_{k-1}^{k} s^{r} A(r, \xi) d r \\
& -\sum_{l=0}^{k-1} \hat{u}^{(l)}(0, \xi) \frac{1}{s^{l+1}} \int_{k-1}^{k} s^{r} A(r, \xi) d r
\end{aligned}
$$

where $\tilde{\hat{u}}(s, \xi)$ is the Laplace transform of $\hat{u}(t, \xi)$. Now summing up by $k$ from 1 till $m$ we obtain

$$
\tilde{\hat{u}}(s, \xi) \int_{0}^{m} A(r ; \xi) s^{r} d r-\sum_{k=0}^{m-1} \frac{\hat{\varphi}_{k}(\xi)}{s^{k+1}} \int_{k}^{m} A(r ; \xi) s^{r} d r=B(\xi) \tilde{\hat{u}}(s, \xi) .
$$

Denote

$$
\Phi_{0}(s, \xi)=0 ; \quad \Phi_{j}(s, \xi)=\int_{0}^{j} s^{r} A(r, \xi) d r, \quad j=1, \ldots, m .
$$

Then it follows from (17) and the hypothesis that only the $j$-th Cauchy datum is non-zero (see (15)) that

$$
\tilde{\hat{u}}_{j}(s, \xi)=\frac{\Phi_{m}(s, \xi)-\Phi_{j}(s, \xi)}{s^{1+j}\left[\Phi_{m}(s, \xi)-B(\xi)\right]} \hat{\varphi}_{j}(\xi), \quad j=0, \ldots, m-1 .
$$

Let $G \subseteq R^{n}$ be an open set and $\xi \in G$ be fixed. Denote by $s_{0}(\xi)$ the greatest positive root of the equation $\Phi_{m}(s, \xi)=B(\xi)$. If $A(r, \xi)$ preserves its sign for every $0<r<m$, then it follows from the inequality $s_{1}^{r}<s_{2}^{r}$ for $0<s_{1}<s_{2}$ and $r>0$ that $\Phi_{m}(s, \xi), s>0$, is a monotone function. Hence, the equation 
$\Phi_{m}(s, \xi)=B(\xi)$ can have no more than one positive root for every fixed $\xi \in G$. Thus

$$
\Psi_{j}(s, \xi)=\frac{\Phi_{m}(s, \xi)-\Phi_{j}(s, \xi)}{s\left[\Phi_{m}(s, \xi)-B(\xi)\right]}
$$

is well defined for $s>s_{0}(\xi)$ if the equation $\Phi_{m}(s, \xi)=B(\xi)$ has a positive root, or for $s>0$, if there is no such a root. It is not difficult to verify that the collection of functions $\Phi_{0}(s, \xi), \ldots, \Phi_{m-1}(s, \xi)$ is linearly independent. Denote by $S_{j}(t, \xi)$ the Laplace preimage of $\Psi_{j}(s, \xi)$, that is

$$
S_{j}(t, \xi)=L^{-1}\left(\frac{\Phi_{m}(s, \xi)-\Phi_{j}(s, \xi)}{s\left[\Phi_{m}(s, \xi)-B(\xi)\right]}\right), \quad j=0, \ldots, m-1 .
$$

Using the theorem on uniqueness of the inverse Laplace transform, we can conclude that the collection $S_{0}(t, \xi), \ldots, S_{m-1}(t, \xi)$ is linearly independent as well. Thus for $u_{j}(t, x)$ we get the representation

$$
u_{j}(t, x)=J^{j} S_{j}(t, D) \varphi_{j}(x),
$$

where $J^{j}$ is the $j$-th power of the common integration operator with lower limit 0 ,

$$
J^{j} f(t)=\underbrace{\int_{0}^{t} \cdots \int_{0}^{\tau}}_{j \text {-times }} f\left(\tau_{1}\right) d \tau_{1}=\int_{0}^{t} \frac{(t-\tau)^{j-1}}{\Gamma(j)} f(\tau) d \tau \text { for } j \geq 1, \quad J^{0}=I,
$$

Here $I$ denotes the identity operator, and $S_{j}(t, D)$ is the pseudo-differential operator with the symbol $S_{j}(t, \xi)$. For the solution of (11), (12) we have the representation

$$
u(t, x)=\sum_{j=0}^{m-1} J^{j} S_{j}(t, D) \varphi_{j}(x) .
$$

Two notes. The obtained representation formula is useful both from mathematical and physical point of views.

1M) The representation is obtained as the action of the pseudo-differential operators $J^{j} S_{j}(t, \xi), j=0, \ldots, m-1$, called $j$-th solution operator, to the given functions. To get solution properties we can use the known properties of these operators. Moreover, all these pseudo-differential operators have a similar structure.

2Ph) The formula says that it does not matter how many different fractional orders are taken in the given equation between two consecutive integers. For the initial value problem it is important to have the initial values of the unknown solution just for integer order derivatives. Moreover, from this formula it can be derived that if the maximal order of the derivatives in the equation is 
not greater than $m-1$, then the Cauchy problem with $m$ given data becomes incorrectly posed. Indeed if $A(r, \xi)=\delta(r-\alpha), \alpha \leq m-1$, rewriting (20) for $j=m-1$ in the form

$$
\Psi_{m-1}(s, \xi)=\frac{\int_{m-1}^{m} s^{r} A(r, \xi) d r}{s\left[\Phi_{m}(s, \xi)-B(\xi)\right]},
$$

we get $S_{m-1}(t, \xi) \equiv 0$.

\section{Some auxiliary lemmas}

Now we give the definition of solution to the Cauchy problem (11), (12).

Definition 4.1. A function $u(t, x)$ is called a strong solution to the Cauchy problem (11), (12) if

1. $u(t, x) \in C^{(m-1)}\left(t \geq 0 ; \Psi_{G, p}\left(\mathbb{R}^{n}\right)\right)$

2. $u^{(m)}(t, x) \in \Psi_{G, p}\left(\mathbb{R}^{n}\right)$ exists for almost all $t>0$ and

3. it satisfies the equation (11) for almost all $t \in(0, \infty)$ and for all $x \in \mathbb{R}^{n}$ including initial conditions (12) pointwise.

Definition 4.2. A function $u(t, x)$ is called a weak solution to the Cauchy problem (11), (12) (replacing $D$ by $-D$ ) if

1. $u(t, x) \in C^{(m-1)}\left(t \geq 0 ; \Psi_{-G, q}^{\prime}\left(\mathbb{R}^{n}\right)\right)$

2. $u^{(m)}(t, x) \in \Psi_{-G, q}^{\prime}\left(\mathbb{R}^{n}\right)$ for almost all $t>0$ and

3. the following equalities hold true for arbitrary $v \in \Psi_{G, p}\left(\mathbb{R}^{n}\right)$ :

$$
\begin{aligned}
\int_{0}^{m}\left\langle D_{*}^{r} u(t, x), A(r, D) v(x)\right\rangle d r & =\langle u(t, x), A(D) v(x)\rangle \quad \text { for a.a. } t \in(0, \infty) \\
\lim _{t \rightarrow+0}\left\langle u^{(k)}(t, x), v(x)\right\rangle & =\left\langle\varphi_{k}(x), v(x)\right\rangle, \quad k=0, \ldots, m-1 .
\end{aligned}
$$

To prove solvability theorems we need some auxiliary lemmas, using the abbreviation

$$
F_{a}(k ; f):=\int_{0}^{a} e^{k t} f(t) d t, \quad k \in R^{1}
$$

where $a$ is a fixed positive real number and $f$ is a generalized function with $\operatorname{supp} f \subset[0, a]$.

\section{Lemma 4.3.}

1. For a regular distribution $f(t)$ with $\operatorname{supp} f \subset[0, d]$,

$$
\left|F_{a}(k ; f)\right|=o\left(e^{d k}\right), \quad k \rightarrow \infty .
$$


2. For a singular generalized function $f(t)$ with $\operatorname{supp} f=\{d\}$,

$$
\left|F_{a}(k ; f)\right|=O\left(e^{(d+\epsilon) k}\right), \quad k \rightarrow \infty,
$$

where $\epsilon>0$ is arbitrarily small.

Proof. Let first $f \in L_{\infty}(0, a),|f(t)| \leq M<\infty$ and supp $f \subset[0, d]$. Then

$$
\left|F_{a}(k ; f)\right|=\left|\int_{0}^{d} e^{k t} f(t) d t\right| \leq M \int_{0}^{d} e^{k t} d t=\frac{M}{k}\left(e^{k d}-1\right) .
$$

For large $k$ we have $\left|F_{a}(k ; f)\right|=o\left(e^{d k}\right)$. For a regular generalized function $f$ with supp $f \subset[0, d]$ there is a sequence of functions $f_{m} \in L_{\infty}(0, a)$, all supported in $[0, d]$ and with common estimation constant $M$ such that $f_{m} \rightarrow f, m \rightarrow \infty$ in the weak sense. For $f_{m}$ we have $k\left|F_{a}\left(k ; f_{m}\right)\right| \leq C e^{k d}$ with positive constant $C$. Letting $m \rightarrow \infty$ we obtain the desired result.

If $f$ is a singular generalized function with support supp $f=\{\alpha\}, 0<\alpha<a$, then $f$ is a finite linear combination of $\delta^{(j)}(t-\alpha)$, where $\delta$ is the Dirac function. It is readily seen that $F_{a}(k ; f)=O\left(e^{(\alpha+\epsilon) k}\right)$ for any $\epsilon>0$ as $k \rightarrow \infty$ in this case.

Lemma 4.4. Let $\Phi_{j}(s, \xi), j=0, \ldots, m$, be as in (18).

1. If $A(r, \xi)$ is a regular distribution with respect to $r$ with $\operatorname{supp} A(r, \xi) \subset$ $[0, d]$, then $\Phi_{j}(s, \xi)=o\left(s^{\nu}\right), s \rightarrow \infty$, where $\nu=\min \{d, j\}$.

2. If $A(r, \xi)$ is a singular distribution with respect to $r$ with $\operatorname{supp} A(r, \xi)=$ $\{d\}$, then $\Phi_{j}(s, \xi)=O\left(s^{d+\epsilon}\right)$, ( $\epsilon$ is arbitrarily small) $s \rightarrow \infty$, in the case $d \leq j$ and $\Phi_{j}(s, \xi)=0$ when $d>j$.

Remark. Hereafter supp $A(r, \xi)$ means the support of $A(r, \xi)$ with respect to the variable $r$.

Proof. We have

$$
\Phi_{j}(s, \xi)=\int_{0}^{j} s^{r} A(r, \xi) d r=\int_{0}^{j} e^{r \ln s} A(r, \xi) d r=: F_{j}(\ln s ; A(r, \xi)), \quad s>s_{0}(\xi) .
$$

Now it is an easy exercise to apply Lemma 4.1 and get the asymptotics in the two cases.

Corollary. For every $j=0, \ldots, m-1, \Psi_{j}(s, \xi)=O\left(\frac{1}{s}\right), s \rightarrow \infty$, where $\Psi_{j}(s, \xi)$ is given by (20).

Lemma 4.5. For every $j=0, \ldots, m-1$, and $k=0,1, \ldots$,

$$
\Phi_{j}^{(k)}(s, \xi)=\frac{1}{s^{k}} \int_{0}^{j} r^{k} s^{r} A(r, \xi) d r=\frac{1}{s^{k}} F_{j}\left(\ln s ; r^{k} A(r, \xi)\right), \quad s>s_{0}(\xi),
$$

where $\xi$ is fixed and derivatives are taken with respect to the variable s. 
Remark. Note that $r$ is a dummy variable in the expressions $F_{j}\left(\ln s ; r^{k} A(r, \xi)\right)$, $k=0,1, \ldots$.

Corollary. For every $j=0, \ldots, m-1, \Psi_{j}^{(k)}(s, \xi)=O\left(\frac{1}{s^{k+1}}\right), s \rightarrow \infty, k \in \mathbf{N}$.

Lemma 4.6. For $S_{j}(t, \xi)=L_{s \rightarrow t}^{-1} \Psi_{j}(s, \xi), j=0, \ldots, m-1$, with any fixed $\xi \in G$ the following assertions are true:

1. $S_{j}(t, \xi) \rightarrow 1$ for $t \rightarrow+0, j=0, \ldots, m-1$;

2. $\frac{\partial^{\ell} S_{j}(t, \xi)}{\partial t^{\ell}} \rightarrow 0$ for $t \rightarrow+0, j=0, \ldots, m-1, \ell=1, \ldots, m-j-1$.

Proof. Let $\xi \in G$ be fixed. It follows from the representation (20) for $\Psi_{j}(s, \xi)$ and Lemma 4.2 that $\Psi_{j}(s, \xi)=O\left(\frac{1}{s}\right)$ for large $s$, which allows to invert $\Psi_{j}(s, \xi)$ (see [37]) with respect to $s$. Thus $S_{j}(t, \xi)$ exists for all $j=0, \ldots, m-1$. Further we use the well known relation [9], [37]

$$
\lim _{s \rightarrow \infty} s \tilde{f}(s)=\lim _{t \rightarrow+0} f(t) .
$$

Lemma 4.2 implies $s \Psi_{j}(s, \xi) \rightarrow 1$ as $s \rightarrow \infty$. Hence $S_{j}(t, \xi) \rightarrow 1$ when $t \rightarrow+0$. We have proved (i). The Laplace transform of $\frac{\partial S_{j}(t, \xi)}{\partial t}$ is

$s \Psi_{j}(s, \xi)-S_{j}(+0, \xi)=\frac{\Phi_{m}(s, \xi)-\Phi_{j}(s, \xi)}{\Phi_{m}(s, \xi)-B(\xi)}-1=\frac{-\Phi_{j}(s, \xi)+B(\xi)}{\Phi_{m}(s, \xi)-B(\xi)}, \quad s>s_{0}(\xi)$,

which is $O\left(\frac{1}{s^{d-j}}\right)$ for large $s$ with $d=\max \operatorname{supp} A(r, \xi)$. Note that $d>m-1$. Otherwise the Cauchy problem is meaningless (see Note $2 \mathrm{Ph})$ ). Analogously for the Laplace transform of $\frac{\partial^{\ell} S_{j}(t, \xi)}{\partial t^{\ell}}$ by induction we get

$$
L\left[\frac{\partial^{\ell} S_{j}(t, \xi)}{\partial t^{\ell}}\right]=s^{l-1}\left(s \Psi_{j}(s, \xi)-S_{j}(+0, \xi)\right)=O\left(\frac{1}{s^{d-j-l+1}}\right) .
$$

Consequently, we have $s L\left[\frac{\partial^{\ell} S_{j}(t, \xi)}{\partial t^{\ell}}\right]=O\left(\frac{1}{s^{d-j-l}}\right)$. Using this and (23) we arrive at (ii).

Lemma 4.7. For every $j=0, \ldots, m-1$ the following assertions hold:

1. $S_{j}(t, \xi) \in C^{m-j-1}(t \geq 0 ; C(G))$.

2. If the upper bound of $\operatorname{supp} A(r, \xi)=m$, then $S_{j}^{(m-j)}(t, \xi)$ exists for almost all $t \in(0, \infty)$.

Proof. In proving the previous lemma we noticed that the $S_{j}(t, \xi), j=0, \ldots$, $m-1$, exist. Now we will check their differentiability properties. It is known [38] that if for given $f(t)$ its Laplace transform $\tilde{f}(s)$ satisfies $s \tilde{f}(s) \rightarrow 0$ when $s \rightarrow \infty$, then $f$ is continuous. For fixed $\xi \in G$ it follows from $(24)$ that

$$
s L\left[\frac{\partial^{\ell} S_{j}(t, \xi)}{\partial t^{\ell}}\right]=O\left(\frac{1}{s^{d-l-j}}\right) .
$$


Take $l=m-j-1$, which means that $s L\left[\frac{\partial^{\ell} S_{j}(t, \xi)}{\partial t^{\ell}}\right]$ vanishes as $|s| \rightarrow \infty$. Hence $\frac{\partial^{\ell} S_{j}(t, \xi)}{\partial t^{\ell}}$ is continuous. Now assume that $d=m$. Then we have $L\left[\frac{\partial^{m-j} S_{j}(t, \xi)}{\partial t^{m-j}}\right]=$ $O\left(\frac{1}{s}\right)$. Thus $\frac{\partial^{m-j} S_{j}(t, \xi)}{\partial t^{m-j}}$ exists for almost every $t \in(0, \infty)$.

Remark. If $m-1<d<m$ one can show that $D_{*}^{d-j} S_{j}(t, \xi), j=0, \ldots, m-1$, exists and is bounded for almost every $t$ (compare with Lemma 7 in [16]).

\section{Solution of the Cauchy problem for distributed order pseudo-differential equations}

In Section 3 the formal representations for a solution of the Cauchy problem (11), (12) have been obtained. In this Section we establish that they represent the strong and weak solutions of the Cauchy problem in the spaces $\Psi_{G, p}\left(\mathbb{R}^{n}\right)$ and $\Psi_{-G, q}^{\prime}\left(\mathbb{R}^{n}\right)$, respectively.

Theorem 5.1. Let $G$ be a domain of continuity of the symbols $A(r, \xi)$ (r fixed) and $B(\xi)$. Let $\varphi_{j} \in \Psi_{G, p}\left(\mathbb{R}^{n}\right), j=0, \ldots, m-1$. Then the Cauchy problem (11), (12) has a unique strong solution. This solution is given by the representation (22).

Proof. Let $G$ be a domain of continuity of the symbols $A(r, \xi)$ and $B(\xi)$ and $\varphi_{j} \in \Psi_{G, p}\left(\mathbb{R}^{n}\right), j=0, \ldots, m-1$. By construction of the representation $(22)$ each of its terms satisfies the equation (11) and, by virtue of Lemma 4.3, the conditions (12). It follows from Lemma 4.4 that for every $j=0, \ldots, m-1$ the inclusion $J^{j} B_{j}(t, \xi) \in C^{m-1}(t \geq 0 ; C(G))$ holds. Moreover, $D_{*}^{d} J^{j} B_{j}(t, \xi)$ is bounded for almost every $t \in(0, \infty)$.

Theorem 5.2. Let $\varphi_{j} \in \Psi_{-G, q}^{\prime}\left(\mathbb{R}^{n}\right), j=0, \ldots, m-1$. Then the Cauchy problem (11), (12) has a unique weak solution given by

$$
u(t, x)=\sum_{j=0}^{m-1} J^{j} S_{j}(t,-D) \varphi_{j}(x),
$$

where $S_{j}(t,-D), j=1, \ldots, m$, is the $j$-th solution operator with symbol $B_{j}(t, \xi)$.

Proof. Let $\varphi_{j} \in \Psi_{-G, q}^{\prime}\left(\mathbb{R}^{n}\right), j=0, \ldots, m-1$. It follows from Theorem 2.1 that every term in the right-hand side of (25), namely, $u_{j}(t, x)=J^{j} S_{j}(t,-D) \varphi_{k-1}(x)$, $j=0, \ldots, m-1$, is a functional from the space $\Psi_{-G, q}^{\prime}\left(\mathbb{R}^{n}\right)$. To prove the theorem we have to show that $u_{j}(t, x), j=0, \ldots, m-1$, satisfies the equation (11) and the initial conditions (12) in the weak sense. Let $v \in \Psi_{G, p}\left(\mathbb{R}^{n}\right)$ be an 
arbitrary function. Then for $u_{j}(t, x)=J^{j} S_{j}(t,-D) \varphi_{j}(x)$ we have

$$
\begin{aligned}
& \int_{0}^{m}\left\langle D_{*}^{r} u_{j}(t, x), A(r, D) v(x)\right\rangle d r-\left\langle u_{j}(t, x), B(D) v(x)\right\rangle \\
& =\left\langle\int_{0}^{m} D_{*}^{r} J^{j} S_{j}(t,-D) \varphi_{j}(x), A(r, D) v(x)\right\rangle d r-\left\langle J^{j} S_{j}(t,-D) \varphi_{j}(x), B(D) v(x)\right\rangle \\
& =\left\langle\varphi_{j}(x),\left[\int_{0}^{m} D_{*}^{r} A(r, D) J^{j} B_{j}(t,-D) d r-B(D) J^{j} S_{j}(t,-D)\right] v(x)\right\rangle .
\end{aligned}
$$

Applying the Fourier transform and remembering the definition of $S_{j}(t, D)$ we conclude that the expression in the square brackets in the last relation is zero for almost every $t \in(0, \infty)$. Besides, Lemma 4.3 yields

$$
\lim _{t \rightarrow+0}\left\langle u_{j}^{(k)}(t, x), v(x)\right\rangle=\left\langle\delta_{j, k} \varphi_{k}(x), v(x)\right\rangle, \quad j, k=0, \ldots, m-1 .
$$

Hence, $u(x, t)$ satisfies the equation (11) in the weak sense.

\section{Solution of the multi-point value problem for distributed order differential equations}

In this section we deal with the general multi-point value problem (5) for the distributed order pseudo-differential equation (3). As we have seen in Section 2 each of the functions $S_{j}(t, \xi), j=0, \ldots, m-1$, satisfies the equation

$$
\int_{0}^{m} A(r ; \xi) D_{*}^{r} S_{j}(t, \xi) d r=B(\xi) S_{j}(t, \xi)
$$

and the initial conditions

$$
\begin{gathered}
S_{j}(0, \xi)=0, \ldots, S_{j}^{j-1}(0, \xi)=0, \\
S_{j}^{j}(0, \xi)=1, \\
S_{j}^{j+1}(0, \xi)=0, \ldots, S_{j}^{m-1}(0, x)=0 .
\end{gathered}
$$

We look for a solution of the multi-point problem in the form

$$
U(t, \xi)=\sum_{j=0}^{m-1} c_{j} S_{j}(t, \xi)
$$

with unknown coefficients $c_{j}=c_{j}(\xi), j=0, \ldots, m-1$. Substituting (26) into

$$
\sum_{j=0}^{m-1} \Gamma_{k j}(\xi) \frac{\partial^{k} U\left(t_{k j}, \xi\right)}{\partial t^{k}}=\hat{\varphi}_{k}(\xi), \quad \xi \in G
$$


which follows from (5) and applying the Fourier transform with respect to $x$ leads to the system of linear algebraic equations

$$
M(\xi) C(\xi)=\hat{\Phi}(\xi) .
$$

Here $M(\xi)$ is a square matrix of order $m$ with the entries

$$
m_{k l}=\sum_{j=0}^{m-1} \Gamma_{k j}(\xi) S_{l}^{(j)}\left(t_{k j}, \xi\right), \quad k, l=0, \ldots, m-1
$$

$C(\xi)=\left(c_{0}(\xi), \ldots, c_{m-1}(\xi)\right)$ is a vector of unknown coefficients and $\hat{\Phi}(\xi)=$ $\left(\hat{\varphi}_{0}(\xi), \ldots, \hat{\varphi}_{m-1}(\xi)\right)$ is the initial data vector. Denote by $M_{0}$ the set of all points $\xi \in G$ such that $\operatorname{Det} M(\xi)=0$. For $\xi \notin M_{0}$ the equation (28) has a unique solution

$$
C(\xi)=M^{-1}(\xi) \hat{\Phi}(\xi) .
$$

We note that $M_{0}$ is the singular set for the symbols of the solution operators. Inserting the representation $(29)$ of the vector $C(\xi)=\left(c_{0}(\xi), \ldots, c_{m-1}(\xi)\right)$ into (26) and applying the inverse Fourier transform we get the solution of the general multi-point value problem (3), (5) as

$$
u(t, x)=\sum_{k=0}^{m-1} U_{k}(t, D) \varphi_{k}(x),
$$

where the $U_{k}(t, D), k=0, \ldots, m-1$, are solution pseudo-differential operators with the symbols $U_{k}(t, \xi)=\left(M^{*}\right)^{-1}(\xi) S(t, \xi)$, the matrix $\left(M^{*}\right)^{-1}(\xi)$ is inverse to the Hermitian conjugate of $M(\xi)$, and $S(t, \xi)$ is the transpose of the row vector $\left(S_{0}(t, \xi), \ldots, S_{m-1}(t, \xi)\right)$ with the components given by $(21)$.

Theorem 6.1. Let $\varphi_{k} \in \Psi_{G^{\prime}, p}\left(\mathbb{R}^{n}\right), k=0, \ldots, m-1, G^{\prime}=G \backslash M_{0}$. Then the multi-point value problem (3), (5) has a unique strong solution in the space $C^{(m)}\left(\left(T_{1}, T_{2}\right) ; \Psi_{G^{\prime}, p}\left(\mathbb{R}^{n}\right)\right)$. This solution is given by the formula (30).

The analogous theorem is valid for the dual problem too. With the dual problem we mean the problem obtained by replacing the operators $A(r, D)$, $B(D)$ and $\Gamma_{k j}(D), j, k=0, \ldots, m-1$, by their dual operators (see formula (10)).

Theorem 6.2. Let $\varphi_{k} \in \Psi_{-G^{\prime}, q}^{\prime}\left(\mathbb{R}^{n}\right), k=0, \ldots, m-1, G^{\prime}=G \backslash M_{0}$. Then the dual multi-point value problem has a unique weak solution in the space $C^{(m)}\left(\left(T_{1}, T_{2}\right) ; \Psi_{-G^{\prime}, q}\left(\mathbb{R}^{n}\right)\right)$. This solution is given by the formula

$$
u(t, x)=\sum_{k=0}^{m-1} U_{k}(t,-D) \varphi_{k}(x) .
$$




\section{Concluding remarks}

Complementing previous works of Umarov and of Umarov et al. (see [16], [33] - [36]) we have elaborated how the Cauchy problem for a general linear evolution equation with (fractional) temporal derivatives of distributed orders can be successfully analyzed by the powerful theory of pseudo-differential operators that has been conceived by Yu. A. Dubinskij in 1982. By applying this theory, using Fourier transform in space, Laplace transform in time, we have solved the questions of strong solutions (in classical function spaces) and weak solutions (in corresponding dual spaces), not only for the Cauchy problem, but also for a rather general multi-point problem. Having in mind applications mainly in physics, where in the case of the Cauchy problem initial conditions are given in form of functions and integer order derivatives we have throughout adhered to the Caputo version of fractional derivatives. Our treatment is constructive, so not only answers the questions for existence and uniqueness but in addition renders transparent integral representations for the solution. For mathematical completeness it would be desirable to treat the analogous problems where instead of the fractional derivatives of Caputo type those of Riemann-Liouville type are used. This, of course, can easily be done in case of all initial conditions assumed to be zero (in this case the two types of fractional derivatives coincde) but seems rather tricky otherwise. We leave this problem to another paper or to other authors.

Acknowledgement.. We have carried out part of this work during the research visit (supported by DAAD, Bonn, Germany) of S. Umarov at the Freie Universität Berlin, Fachbereich Mathematik und Informatik, Institut für Mathematik I, in December 2003 and January 2004. We gratefully acknowledge the good working conditions offered there. We appreciate the careful work of the anonymous referees with our paper and their valuable suggestions for improvement.

\section{References}

[1] Antipko, I. I. and V. M. Borok: The Neumann boundary value problem in an infinite layer. J. Sov. Math. 58 (1992), 541 - 547. (Translation from Theoret. Funktion. Anal. Prilozh. 53 (1990), $71-78$.)

[2] Bagley, R. L. and P. J. Torvik: On the existence of the order domain and the solution of distributed order equations. Part 1, 2. Int. J. Appl. Math. 2 (2000)(7), $865-882$, and (2000)(8), $965-987$.

[3] Borok, V. M.: Correctly solvable boundary-value problems in an infinite layer for systems of linear partial differential equations. Math. USSR, Izv. 5 (1971), $193-210$.

[4] Caputo, M.: Linear models of dissipation whose $Q$ is almost frequency independent. Part 2. Geophys. J. R. Astr. Soc. 13 (1967), 529 - 539. 
[5] Caputo, M.: Mean fractional order derivatives. Differential equations and filters. Annals Univ. Ferrara - Sez. VII - SC. Mat., Vol. XLI (1995), 73 - 84.

[6] Caputo, M.: Distributed order differential equations modeling dielectric induction and diffusion. Fract. Calc. Appl. Anal. 4 (2001), 421 - 442.

[7] Chechkin, A. V., Gorenflo, R., Sokolov, I. M. and V. Yu. Gonchar: Distributed order time fractional diffusion equation. Fract. Calc. Appl. Anal. 6 (2003), 259 -279 .

[8] Diethelm, K. and N. J. Ford: Numerical solution methods for distributed order time fractional diffusion equation. Fract. Calc. Appl. Anal. 4 (2001), 531 - 542.

[9] Ditkin, V. A. and A. P. Prudnikov: Integral Transforms and Operational Calculus. Oxford: Pergamon 1965.

[10] Dubinskij, Yu. A.: On a method of solving partial differential equations. Sov. Math. Dokl. 23 (1981), 583 - 587.

[11] Dubinskij, Yu. A.: The algebra of pseudo-differential operators with analytic symbols and its applications to mathematical physics. Soviet Math. Survey 37 (1982), $107-153$.

[12] Gorenflo, R. and F. Mainardi: Fractional calculus: integral and differential equations of fractional order. In: Fractals and Fractional Calculus in Continuum Mechanics (Udine 1996), (eds.: A. Carpinteri and F. Mainardi). Wien: Springer 1997, pp. $223-276$.

[13] Gorenflo, R. and F. Mainardi: Random walk models for space-fractional diffusion processes. Fract. Calc. Appl. Anal. 1 (1998), 167 - 191.

[14] Gorenflo, R. and F. Mainardi: Fractional calculus and stable probability distributions. Archives of Mechanics 50 (1998), 377 - 388.

[15] Gorenflo, R. and F. Mainardi: Approximation of Lévy-Feller diffusion by random walk. Z. Anal. Anw. 18 (1999), 231 - 246.

[16] Gorenflo, R., Yu. Luchko and S. Umarov: The Cauchy and multi-point partial pseudo-differential equations of fractional order. Fract. Calc. Appl. Anal. 3 (2000)(3), $249-275$.

[17] Gorenflo, R. and A. Vivoli: Fully discrete random walks for space-time fractional diffusion equations. Signal Processing 83 (2003), 2411 - 2420.

[18] Hoh, W.: Pseudo differential operators with negative definite symbols of variable order. Rev. Mat. Iberoam., 16 (2000)(2), 219 - 241.

[19] Jacob, N. and H-G. Leopold: Pseudo differential operators with variable order of differentiation generating Feller semigroups. Integr. Equat. Oper. Theory 17 (1993)(4), $544-553$.

[20] Jacob, N. and A. Krägeloh: The Caputo derivative, Feller semigroups, and the fractional power of the first order derivative on $C_{\infty}\left(R_{0}^{+}\right)$. Fract. Calc. Appl. Anal. 5 (2002)(4), 395 - 410.

[21] Kochubei, A.: Parabolic pseudodifferential equations, hypersingular integrals and Markov processes (in Russian). Math. USSR Izv. 33 (1989)(2), 233 - 259. 
[22] Lorenzo, C. F. and T. T. Hartley: Variable order and distributed order fractional operators. Nonlinear Dynam. 29 (2002), 57 - 98.

[23] Mainardi, F.: Fractional calculus: some basic problems in continuum and statistical mechanics. In: Fractals and Fractional Calculus in Continuum Mechanics (Udine 1996), (eds.: A. Carpinteri and F. Mainardi). Wien: Springer 1997, pp. $291-348$.

[24] Meerschaert, M. and D. Benson: Governing equations and solutions of anomalous random walk limits. Physical Review E, 060102(R), 66 (2002), 1 - 4.

[25] Meerschaert, M. and H-P. Scheffler: Limit theorems for continuous time random walks with slowly varying waiting times. Statistics and Probability Letters 71 (2005)(1), $15-22$.

[26] Metzler, R. and J. Klafter: The random walk's guide to anomalous diffusion: a fractional dynamics approach. Physics Reports 339 (2000), 1 - 77.

[27] Podlubny, I.: Fractional Differential Equations. Mathematics in Science and Engineering Vol. 198. San Diego (CA): Academic Press 1999.

[28] Ptashnik, B. I.: Ill-Posed Boundary Value Problems for Partial Differential Equations (in Russian). Kiev: Naukova Dumka 1984.

[29] Samko, S. G., Kilbas, A. A. and O. I. Marichev: Fractional Integrals and Derivatives: Theory and Applications. New York: Gordon and Breach 1993.

[30] Sokolov, I. M., Chechkin, A. V. and J. Klafter: Distributed-order fractional kinetics. Acta Physica Polonica B, 35 (2004), 1323 - 1341.

[31] Schneider, W. R. and W. Wyss: Fractional diffusion and wave equations. J. Math. Physics 30 (1989), 134 - 144.

[32] Tran Duc Van: On the pseudo-differential operators with real analytic symbol and their applications. J. Fac. Sci. Univ. Tokyo, IA, Math. 36 (1989), 803 825.

[33] Umarov, S. R.: Boundary value problems for differential operator and pseudodifferential equations (in Russian). Izv. Acad. Sci. RU 4 (1986), 38 - 42.

[34] Umarov, S. R.: Nonlocal boundary value problems for pseudo-differential and differential operator equations I. Differ. Equations 33 (1997), 831 - 840.

[35] Umarov, S. R.: Nonlocal boundary value problems for pseudo-differential and differential operator equations II. Differ. Equations 34 (1998), 374 - 381.

[36] Umarov, S. R. and R. Gorenflo: On multi-dimensional random walk models approximating symmetric space-fractional diffusion processes. Fract. Calc. Appl. Anal. (to appear).

[37] Widder, D. V.: The Laplace Transform. Princeton: University Press 1946.

[38] Zemanian, A. H.: Generalized Integral Transformations. New York: Wiley 1968.

Received 06.09.2004 